\title{
HIDRÓLISE ENZIMÁTICA, FERMENTAÇÃO E PRODUÇÃO DE BIOCOMBUSTÍVEIS ATRAVÉS DA COROA DE Ananas comosus
}

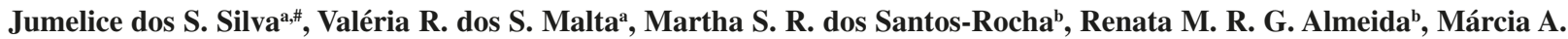
Gomes $^{c}$, Cecília D. Vicente ${ }^{\mathrm{d}}$ e Kledson L. Barbosa ${ }^{\mathrm{a}, *}$

Instituto de Química e Biotecnologia, Universidade Federal de Alagoas, 57072-900 Maceió - AL, Brasil

${ }^{\mathrm{b} C e n t r o ~ d e ~ T e c n o l o g i a, ~ U n i v e r s i d a d e ~ F e d e r a l ~ d e ~ A l a g o a s, ~ 57072-900 ~ M a c e i o ́ ~-~ A L, ~ B r a s i l ~}$

'Departamento de Engenharia Industrial, Universidade Federal da Bahia, 40210-630 Salvador - BA, Brasil

${ }^{\mathrm{d}}$ Departamento de Química, Instituto Federal de Alagoas, 57035-660 Maceió - AL, Brasil

Recebido em 26/04/2017; aceito em 07/08/2018; publicado na web em 29/08/2018

\begin{abstract}
ENZYMATIC HYDROLYSIS, FERMENTATION AND BIOFUELS PRODUCTION FROM ANANAS COMOSUS CROWN. Problems related to the use of fossil fuels and greenhouse gas emissions have become global concerns. To address this issue, the strategy adopted in the present study was to use Ananas comosus residues as a source of fermentable carbohydrates to produce second generation ethanol and briquettes. The demand for biofuel is addressed in the present study by the integrated processing of several stages of treatment of the lignocellulosic substrate: chemical characterization, pretreatment, enzymatic hydrolysis and fermentation. It was verified under the conditions used that the enzymatic hydrolysis liberated after 72 hours 58.44 and $21.91 \mathrm{~g} \mathrm{~L}^{-1}$ of glucose and xylose, respectively. Conversion of the sugars in the fermentation step resulted in $18.80 \mathrm{~g} \mathrm{~L}^{-1}$ ethanol in 24 hours. The briquettes produced from the remaining solid fraction of the enzymatic hydrolysis presented a calorific value of $18.41 \mathrm{~kJ} \mathrm{~kg}^{-1}$.
\end{abstract}

Keywords: briquettes; ethanol 2G; enzymatic hydrolysis; fermentation; pretreatment.

\section{INTRODUÇÃo}

A rápida expansão da população humana mundial, o aumento da frequência e intensidade de regiões secas, e a diminuição de umidade do solo, tornaram-se os principais problemas da sociedade no século XXI. ${ }^{1-3}$ Esses agravos comprometem a segurança alimentar e o futuro energético, uma vez que aumenta-se a disputa por recursos hídricos e terrestres, sejam na produção urbana ou na agricultura, exigindo cada vez mais o uso de água doce. ${ }^{4}$ No entanto, a existência e o desenvolvimento de novas tecnologias buscam mitigar esses desafios através do uso de plantas com metabolismo especializado de fotossíntese, que as tornam capazes de suportar o estresse hídrico em regiões de seca. ${ }^{5}$ Essa direção positiva para tais plantas as tornam alvo para o estudo de culturas

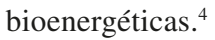

O progresso no uso de plantas com metabolismo ácido crassuláceo (CAM) pode ser alcançado através da transferência genética das características das plantas CAM para as culturas de alimentos e de biomassa já existentes. Algumas espécies de plantas CAM já estão sendo investigadas para a produção de biocombustíveis devido a seu rendimento teórico de biomassa, como, por exemplo, Agave spp. ${ }^{6}$ Opuntia ficus-indica ${ }^{7}$ e Sisal spp. ${ }^{8}$ Outro ponto importante é que com o uso de plantas CAM, podem-se alcançar rendimentos maiores de colheita em regiões de intenso calor e seca, além de contribuir com a possibilidade de utilizar terras abandonadas e semiáridas para a produção agrícola. ${ }^{4}$

Considerada uma alternativa à produção de biocombustível, a biomassa lignocelulósica trata-se de uma fonte de energia renovável de baixo custo, com grande disponibilidade geográfica e com redução líquida nas emissões de carbono na atmosfera. ${ }^{9-11}$ A biomassa pode ser processada em diferentes equipamentos e apresentar diferentes

*e-mail: kledsonlopesb@gmail.com

"e-mail alternativo: jumelicequimica@gmail.com tamanhos de partículas. Em uma usina de energia, por exemplo, os pellets de biomassa são utilizados sob a forma de pequenas partículas, o que permite seu uso como carvão. ${ }^{12,13}$

A biotransformação dos resíduos agrícolas em açúcares fermentescíveis com posterior produção de etanol de segunda geração é economicamente viável. Entretanto, as tecnologias empregadas para bioconversão da biomassa lignocelulósica, através dos resíduos agroindustriais ainda não são totalmente eficientes para produção de etanol em escala industrial, haja vista os custos com capital e operacionais. ${ }^{14,15}$ Mesmo com esses desafios, as fontes de energias renováveis continuam sendo uma alternativa para substituir gradualmente o uso de combustíveis fósseis. Além disso, é importante para a geração de empregos nas áreas rurais e pode substituir a importação de combustível refinado. ${ }^{16-18}$

Nesse sentido, o presente estudo teve como objetivo produzir etanol de segunda geração a partir da coroa do Ananas comosus (parte superior do fruto) utilizando etapas integradas de pré-tratamento à base de água em temperaturas moderadas, hidrólise enzimática e fermentação. Além disso, o trabalho também teve o objetivo de propor o uso dos resíduos remanescentes da hidrólise enzimática para gerar combustível sólido na forma de briquetes.

\section{PARTE EXPERIMENTAL}

\section{Materiais}

Os resíduos de Ananas comosus foram obtidos a partir de um mercado local em Maceió - Alagoas. Para fins analíticos, foi utilizado neste estudo apenas a coroa do abacaxi (parte superior do fruto). A caracterização química foi realizada de acordo com o procedimento analítico descrito por Sluiter et al. ${ }^{19}$ modificado por Rocha et al. ${ }^{20} \mathrm{e}$ validado por Gouveia et al. ${ }^{21}$

Os ensaios foram realizados no Laboratório de Bebidas e Tecnologia de Alimentos, localizado no Centro de Tecnologia da Universidade Federal de Alagoas, durante o ano de 2017. 


\section{Pré-tratamento}

Para o pré-tratamento dos resíduos de Ananas comosus, $10 \mathrm{~g}$ de biomassa foram suspensas em $90 \mathrm{~mL}$ de água destilada em frascos de Erlenmeyer em reator do tipo autoclave a $121^{\circ} \mathrm{C}$ por 30 minutos de reação. Depois de retornar à temperatura ambiente, as misturas foram filtradas para recuperação da fração sólida. No final do procedimento, a fração sólida foi seca em estufa a $50{ }^{\circ} \mathrm{C}$ até atingir um teor de umidade abaixo de $10 \%$, e então armazenada à temperatura ambiente para uso posterior na etapa de hidrólise enzimática.

\section{Hidrólise enzimática}

A biomassa pré-tratada foi hidrolisada com uma carga de sólidos de $15 \%(\mathrm{p} / \mathrm{v})$, equivalente a 7,5 g de biomassa seca, utilizando 20 FPU/g de biomassa do complexo enzimático Cellic ${ }^{\circledR}$ CTec2Cellulase (Novozymes Latin America Ltda) em meio reacional de $50 \mathrm{~mL}$ contendo tampão citrato $50 \mathrm{mmol} \mathrm{L}^{-1}$ (pH 5,0). Os ensaios foram incubados em um agitador a $50^{\circ} \mathrm{C}, 150 \mathrm{rpm}$ por 24 horas, com retiradas de alíquotas nos tempos de 0, 6, 12, 24, 48 e 72 horas para quantificar os açúcares liberados por cromatografia líquida de alta eficiência (HPLC). A atividade enzimática do complexo celulolítico foi determinada de acordo com Ghose. ${ }^{22}$

\section{Preparação de inóculo de levedura}

A reativação da levedura Saccharomyces cerevisiae JAY-270 foi realizada suspendendo as células cerca de $0,1 \%$ em $100 \mathrm{~mL}(\mathrm{v} / \mathrm{v})$ de meio de cultura líquido YEPD (1\% de extrato de levedura; $1 \%$ de peptona de carne e $2 \%$ dextrose) e incubou-se a $28^{\circ} \mathrm{C}$ por 48 horas a $100 \mathrm{rpm}$. Após o período de incubação, o meio de cultura foi centrifugado para retirada da biomassa de levedura.

\section{Fermentação do licor sacarificado}

Inoculou-se 5\% de cultura de células em $20 \mathrm{~mL}$ do licor hidrolisado de Ananas comosus (v/v), suplementado com extrato de levedura (6,8 g L $\left.{ }^{-1}\right), \operatorname{MgSO}_{4}\left(1,4 \mathrm{~g} \mathrm{~L}^{-1}\right)$, uréia $\left(5,3 \mathrm{~g} \mathrm{~L}^{-1}\right)$ e $\mathrm{KH}_{2} \mathrm{PO}_{4}\left(5,6 \mathrm{~g} \mathrm{~L}^{-1}\right)$. As fermentações foram realizadas em um agitador a $30^{\circ} \mathrm{C}$ e $150 \mathrm{rpm}$ por 24 horas. Em seguida, amostras foram coletadas nos tempos de 0 , 2, 4, 8 e 24 horas para monitorar a cinética do consumo de açúcares e a produção de etanol por HPLC.

\section{Produção de briquetes}

Esta etapa foi realizada na mini Usina de adensamento de biomassa da Universidade Federal de Alagoas - UFAL, fazendo uso da prensa IKA-WERKE, com $0,5 \mathrm{~g}$ do material, sob pressão por 3 minutos. Em seguida, foram realizadas análises físico-químicas de poder calorífico, umidade e teor de cinzas.

\section{Análise estatística}

Os ensaios foram realizados usando-se três réplicas para cada amostra. Os dados são expressos como a média da triplicata \pm 0 desvio padrão. Para análise estatística utilizou-se o software Origin versão 8.0.

\section{RESULTADOS E DISCUSSÃO}

\section{Caracterização química}

A composição química média da coroa de Ananas comosus não tratada aplicada neste estudo é apresentada na Tabela 1.

Tabela 1. Propriedades químicas e estruturais dos resíduos de Ananas comosus

\begin{tabular}{lc}
\hline Componentes $(\%)$ & Teor obtido em 2 g de biomassa seca \\
\hline Celulose $^{*}$ & $20,57 \pm 1,17$ \\
Hemicelulose* & $15,24 \pm 0,90$ \\
Lignina solúvel & $7,30 \pm 0,55$ \\
Lignina insolúvel & $19,47 \pm 1,15$ \\
Lignina Total & $26,77 \pm 0,87$ \\
Cinzas & $5,73 \pm 0,22$ \\
\hline Total & 68,32 \\
\hline
\end{tabular}

*Análises determinadas em HPLC. Todas as análises foram realizadas em triplicata.

Huang et al. ${ }^{23}$ também determinaram as propriedades físico-químicas das fibras das cascas do abacaxi como um ingrediente potencial. Os autores mostraram que os principais açúcares presentes na biomassa seca eram glicose e xilose, afirmando ainda que os resíduos do abacaxi são formados majoritariamente por celulose. Considerando o balanço de massa referente a caracterização química da biomassa, é possível constatar que apenas $68,32 \%$ da composição do material lignocelulósico de Ananas comosus foi determinada. Possivelmente, a porcentagem de $31,68 \%$ esteja relacionada com outros componentes estruturais, como proteínas, lipídeos e outros açúcares não quantificados nesse estudo.

Com o objetivo de introduzir um novo processo para produção de etanol, Pourbarani et $a l .{ }^{24}$ registraram também a composição química do resíduo de laranja, demonstrando que esse substrato cítrico continha em sua composição $22 \%$ de celulose, $11,09 \%$ de hemicelulose e $2,19 \%$ de lignina. Comparando a composição estrutural desse resíduo com o de Ananas comosus, pode-se afirmar que a porcentagem dos complexos poliméricos (celulose e hemicelulose) foi bem próxima a dos resultados descritos neste estudo. Contudo, uma expressiva diferença para a fração lignina, provavelmente, por ser um dos principais constituintes da planta, sendo responsável pela sua resistência, ${ }^{25}$ característica encontrada nas folhas de Ananas comosus, que compõe a coroa. Outra possível explicação pode estar relacionada com sua menor solubilidade o que faz com que esse polímero não seja facilmente digerido.

\section{Hidrólise enzimática da coroa de Ananas comosus}

Na Figura 1 é mostrada a biotransformação da biomassa lignocelulósica através da hidrólise enzimática. Após quantificação em HPLC, 58,44 e $21,91 \mathrm{~g} \mathrm{~L}^{-1}$ de glicose e xilose, respectivamente, foram alcançados.

Os resultados obtidos nesse estudo afirmam, portanto, que a hidrólise da coroa de Ananas comosus, utilizada como substrato, teve impacto positivo no metabolismo enzimático para a produção de açúcares fermentescíveis. Além disso, é possível deduzir que a atividade enzimática teve crescimento exponencial à medida que o tempo de hidrólise enzimática prosseguia, mostrando que esse processo resultou em diminuição das fibras de biomassa e um aumento concomitante dos açúcares solúveis, porém, com menor taxa de liberação para xilose.

Para os carboidratos totais quantificados nesse estudo (Tabela 1), o teor de glicose foi $37,47 \%$ maior no licor sacarificado em relação à liberação de xilose, o que pode representar uma hidrólise mais eficiente para conversão de celulose em glicose, visto que o complexo enzimático utilizado caracteriza-se por conter em sua formulação 


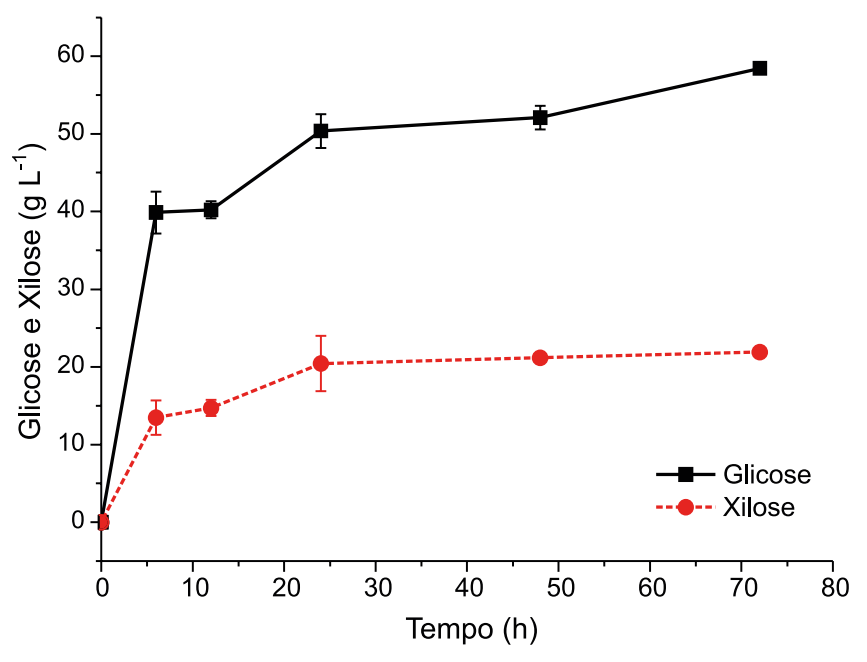

Figura 1. Biotransformação dos resíduos da coroa de Ananas comosus durante a hidrólise enzimática (os valores são dados em $\left.g_{\text {açúcar }} / L_{\text {solução }}\right)$

celulases. Além disso, esses resultados foram obtidos com uma carga enzimática de $20 \mathrm{FPU} / \mathrm{g}$ de biomassa, sugerindo, portanto, ser um dado potencial para aplicação futura de novos ensaios de hidrólise enzimática com diferentes cargas de sólidos e enzima, assim como, testes de hidrólise combinada empregando-se celulases e hemicelulases na mesma reação.

Por outro lado, ainda que a condição de hidrólise tenha sido favorável à celulose, a concentração de xilose alcançada $\left(21,91 \mathrm{~g} \mathrm{~L}^{-1}\right)$ mostrou-se bastante significativa, levando-se a considerar a importância do uso de leveduras recombinantes, capazes de fermentar simultaneamente glicose e xilose, visando uma produção de etanol mais atrativa. Segundo Tropea et al., ${ }^{26}$ essa alternativa de uso de microrganismos melhorados geneticamente pode valorizar as concentrações e a produtividade finais de etanol, uma vez que as pentoses (majoritariamente xilose) poderiam ser também fermentadas a partir do licor sacarificado.

Ainda sobre a redução de açúcares da biomassa da coroa de Ananas comosus, Boonwong et al. ${ }^{27}$ investigaram o seu potencial para produção de açúcares por via enzimática. Após o pré-tratamento da biomassa empregando o ácido sulfúrico como catalisador da reação para desestruturar as fibras da coroa do abacaxi, os autores empregaram a enzima cellulase (Cellic $\left.{ }^{\circledR C T e c} 2\right)$ na etapa de hidrólise enzimática obtendo-se $113,70 \mathrm{~g} \mathrm{~L}^{-1}$ de açúcares redutor. No entanto, esses resultados apontam fragilidades no que se refere a quantificação específica dos diferentes carboidratos presentes nas frações celulose e hemicelulose da biomassa, uma vez que não houve, por exemplo, distinção das hexoses e pentoses. A fermentação dos açúcares redutores totais com a cepa de Saccharomyces cerevisiae TISTR 5596 alcançou a produção máxima de $33,58 \mathrm{~g} \mathrm{~L}^{-1}$ de etanol, demonstrando que os resíduos da coroa do abacaxi podem ser um material adequado para produção de etanol.

\section{Fermentação do licor hidrolisado de Ananas comosus}

Na Figura 2 é mostrado o curso da produção de etanol bem como o consumo dos açúcares presentes na biomassa. A taxa de utilização de açúcares pela levedura Saccharomyces cerevisiae JAY-270 nas quatro primeiras horas foi relativamente alta e a produção de etanol apresentou considerável declínio após 5 horas de fermentação. A produção de etanol foi de $18,80 \mathrm{~g} \mathrm{~L}^{-1}$ em 24 horas, porém, seu mais alto grau foi obtido após as primeiras 4 horas de fermentação alcançando $31,10 \mathrm{~g} \mathrm{~L}^{-1}$ de etanol. A produção de etanol a partir dos resíduos das folhas de Ananas comosus foi investigada por Chintagunta et al. ${ }^{28} \mathrm{em}$ um estudo descrito na literatura e, para isto, utilizaram-se estratégias de hidrólise enzimática e fermentação simultânea. Os autores alcançaram produção de etanol de 7,12\% (v/v) em 24 horas de fermentação, o que reforça a valorização dos resíduos das folhas de Ananas comosus obtidos dos setores agrícola e agroindustrial.

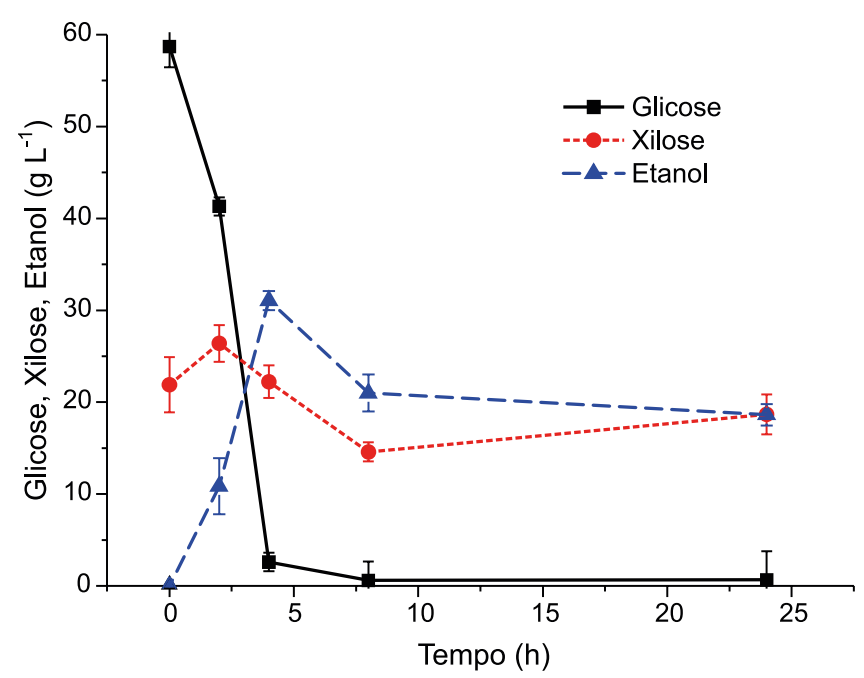

Figura 2. Consumo dos açúcares e produção de etanol ao longo da fermentação etanólica

Bhandari et $a l .{ }^{29}$ estudaram a produção de etanol celulósico a partir das cascas de frutas Ananas comosus em um processo de fermentação sem aeração e revelaram rendimento de etanol de $4,38 \mathrm{~g} \mathrm{~L}^{-1}$, com percentual de conversão de $84,2 \%$. Esses resultados apresentam um futuro promissor para o reaproveitamento desses resíduos celulósicos para a produção de etanol. Embora esse achado tenha sido inferior ao alcançado no presente estudo $\left(18,80 \mathrm{~g} \mathrm{~L}^{-1}\right.$ etanol) utilizando a coroa de Ananas comosus.

O estudo da produção simples e aprimorada de etanol lignocelulolítico por hidrólise ácida sobre os resíduos das cacas de Ananas comosus foi investigado por Niwaswong et al. ${ }^{30}$ os resultados indicaram que o caldo fermentado com a levedura Saccharomyces cerevisiae TISTR 5048 produziu etanol com rendimento de $65,27 \pm 2,45 \%$, relatando um processo de baixo custo para as indústrias. No entanto, a hidrólise ácida apresenta certa desvantagem, uma vez que propicia a formação de inibidores que afetam o metabolismo das leveduras e como consequência, diminui o rendimento etanólico, sendo necessário avaliar o pré-tratamento mais adequado a fim de evitar tais interferentes.

Experimentos para produção de etanol a partir dos resíduos da folha de abacaxi com etapas integradas de sacarificação enzimática (celulase e xilanase) e fermentação simultânea alcançaram produção máxima de etanol de 7,12\% (v/v) em 24 h de fermentação com taxa de conversão de $75,53 \%$. Estabelecendo desta forma, um processo valioso para geração de energia para atender os problemas abordados sobre a crise energética por meio deste e do nosso estudo que utilizou os resíduos lignocelulósicos de Ananas comosus como substrato rico em polissacarídeos para conversão em etanol. ${ }^{31}$

Gil e Maupoey ${ }^{32}$ propuseram um processo de otimização da produção de etanol por meio de diferentes etapas de processamento da biomassa de Ananas comosus, dentre estas: fermentação do licor hidrolisado, sacarificação e fermentação consecutiva dos resíduos sólidos, e sacarificação e fermentação simultânea. Os resultados apontaram a sacarificação e fermentação simultânea como a melhor condição de processamento da biomassa, uma vez que a produção de etanol foi de $5,4 \%(v / v)$, enquanto que com a fermentação direta do hidrolisado obteve-se $4,7 \%$ e com a sacarificação e fermentação dos resíduos sólidos a produção de etanol foi de $4,9 \%$. Sendo assim, 
esses resultados indicam que a sacarificação e fermentação simultânea pode ser uma condição a ser adotada para a fermentação da biomassa de Ananas comosus, visto que proporcionou melhor rendimento de etanol tendo um direcionamento positivo para diminuir os custos com o uso de enzimas celulolíticas.

Verifica-se que nas condições empregadas, a fermentação deveria encerrar-se nas 4 primeiras horas (Figura 2), visto que após esse tempo houve redução no teor de etanol produzido. Sugere-se que, na falta da fonte primária de carbono (glicose), a levedura passe a consumir o etanol. Desta forma, esse mecanismo torna-se economicamente inviável, visto que o objetivo da fermentação é a obtenção de etanol, e seu consumo é indesejado devendo-se, portanto, encerrar a reação de fermentação antes que isso ocorra. Outro artifício a ser considerado é a suplementação de diferentes fontes de nitrogênio para aumentar a biomassa de leveduras, ${ }^{24}$ a partir das quais espera-se acelerar a conversão de açúcares para etanol.

De acordo com essas observações, o inóculo da levedura fez com que o conteúdo de carboidratos fermentescíveis diminuissem de 58,44 para $0,67 \mathrm{~g} \mathrm{~L}^{-1}$, no caso da glicose e de 21,91 para $18,83 \mathrm{~g} \mathrm{~L}^{-1}$, no caso da xilose, representando $87,22 \%$ e $1,16 \%$ de consumo, respectivamente. No final da fermentação, a xilose residual representa, presumivelmente, a atividade enzimática remanescente no licor sacarificado. Após 2 horas de adição da levedura, a xilose apresentou incremento em razão das outras amostragens.

\section{Produção de briquetes}

Os briquetes produzidos a partir do resíduo sólido da hidrólise enzimática apresentaram poder calorífico de $18,41 \mathrm{~kJ} \mathrm{~kg}^{-1}, 11,64 \%$ de umidade e 5,94\% de cinzas. Considerando seu potencial energético, o resíduo sólido remanescente do processo de produção de etanol de segunda geração torna-se, portanto, um substrato complementar para combustão direta em fornos industriais em substituição a lenha. Além disso, o valor de aquecimento é um fator de qualidade importante para a combustão, assim como relataram Demirbas e Sahin. ${ }^{33}$ Sendo assim, os testes de laboratório mostraram que os briquetes produzidos podem complementar o processo de combustão, tornando-se combustíveis cujas perspectivas parecem ser boas para aplicação relacionada ao calor em pequenas indústrias.

As análises complementares no estudo da produção de combustível sólido (briquetes) incluíram a produção de cinzas e umidade. Quanto ao teor de cinzas, sabe-se que este componente representa os óxidos inorgânicos que permanecem após a combustão completa dos materiais. ${ }^{34}$ Os briquetes produzidos neste estudo apresentaram menor teor de cinzas $(5,4 \%)$ quando comparado com outro estudo descrito na literatura, sobre a viabilidade de produção de briquetes através da mistura de vários resíduos de biomassa, em que o teor de cinzas variou entre 14,6 e 31\%. ${ }^{35}$ Quanto ao teor de umidade, sabe-se que este parâmetro pode influenciar no valor do aquecimento do material. ${ }^{36}$ No entanto, não avaliamos neste estudo se um menor ou maior teor de umidade poderia causar alterações no valor de aquecimento do briquete. Outras condições de fabricação, tais como a temperatura e pressão também são responsáveis por influenciar o poder calorífico. ${ }^{37,38}$

Ademais, outro ponto positivo para a produção de briquetes neste estudo está relacionado com a natureza homogênea do resíduo lignocelulósico de Ananas comosus utilizado, em que se pode supor que o material forma uma ligação mais forte, tornando o briquete mais denso e estável. ${ }^{39}$ Combustível sólido como serragem, briquete de biocarvão, lenha e carvão, apresentam valor de aquecimento em torno de 18,65 a 27,98 MJ kg-1 (8.000 a $12.000 \mathrm{Btu} \mathrm{lb}^{-1}$ ). Já o carvão betuminoso, o mais utilizado nas indústrias, apresenta variações entre 24,48 e $36,14 \mathrm{MJ} \mathrm{kg}^{-1}$ (10.500 a $\left.15.500 \mathrm{Btu} \mathrm{lb}^{-1}\right) .^{40}$ Isso implica em dizer que a tecnologia de briquetagem empregada neste estudo, considerando o reaproveitamento de resíduos sólidos de Ananas comosus e o padrão de baixo custo de produção, tornou possível a produção de combustível sólido para atender, ainda que de forma não consolidada, a complementariedade das demandas energéticas de pequenos produtores.

\section{CONCLUSÃO}

Através dos ensaios de hidrólise enzimática realizados no presente trabalho, é possível destacar a importância da aplicação de enzimas celulolíticas sobre o resíduo lignocelulósico de Ananas comosus a fim de obter maiores concentrações de açúcares no licor sacarificado e, por conseguinte, maior rendimento etanólico na etapa de fermentação. Conforme verificado no perfil de hidrólise, a enzima empregada não sofreu perdas de sua atividade catalítica pelos parâmetros utilizados, a $50{ }^{\circ} \mathrm{C}, 150 \mathrm{rpm}, 24$ horas. Deve-se ressaltar também que o uso combinado de enzimas celulases e hemicelulases é um processo que merece ser explorado, no sentido de aumentar as concentrações de açúcares fermentescíveis e maior reaproveitamento dessa biomassa.

De fato, os resultados da fermentação etanólica do licor sacarificado foram dados adicionais para elucidar a configuração dos processos de fermentação, tendo como base a utilização de substratos lignocelulósicos de Ananas comosus através da integração de pré-tratamentos a base de água em temperaturas moderadas e tratamento enzimático. Isso significa dizer que este artigo apresenta dados primários que garantem a redução do tempo no processo de produção de etanol de segunda geração, assim como, melhorias futuras nas etapas experimentais que podem acarretar em menores custos operacionais.

A incorporação do resíduo da biomassa remanescente da hidrólise enzimática para produção de briquetes (obtido dos mercados alagoanos) proporciona uma oportunidade de alavancar estudos nessa perspectiva, uma vez que essa integração ainda é incipiente. Ademais, essa nova aplicabilidade da biomassa destaca seu potencial, dada a sua versatilidade capaz de gerar duas formas de energia (líquida e sólida). A tecnologia empregada para briquetagem é de fácil operação e de baixo custo, o que torna o briquete produzido nesse estudo uma fonte renovável de combustível sólido com boa relação custo-benefício.

\section{REFERÊNCIAS}

1. Gerland, P.; Raftery, A. E.; Sevcikova, H.; Li, N.; Gu, D.; Spoorenberg, T.; Alkema, L.; Fosdick, B. K.; Chunn, J.; Lalic, N.; Bay, G.; Buettner, T.; Heilig, G. K.; Wilmoth, J.; Science 2014, 346, 234.

2. Dai, A.; Nat. Clim. Change 2013, 3, 52.

3. Cook, B.; Smerdon J.; Seager, R.; Coats, S.; Climate Dynamics 2014, $43,1$.

4. Yang, X.; Cushman, J. C.; Borland, A. M.; Edwards, E. J.; Wullschleger, S. D.; Tuskan, G. A.; Owen, N. A.; Griffiths, H.; Smith, J. A. C.; Paoli, H. C. D.; Weston, D. J.; Cottingham, R.; Hartwell, J.; Davis, S. C.; Silvera, K.; Ming, R.; Schlauch, K.; Abraham, P.; Stewart , J. R.; Guo, H. B.; Albion, R.; Ha, J.; Lim, S. D.; Wone, B. W.; Yim, W. C.; Garcia, T.; Mayer, J. A.; Petereit, J.; Nair, S. S.; Casey, E.; Hettich, R. L.; Ceusters, J.; Ranjan, P.; Palla, K. J.; Yin, H.; Reyes-García, C.; Andrade, J. L.; Freschi, L.; Beltrán, J. D.; Dever, L. V.; Boxall, S. F.; Waller, J.; Davies, J.; Bupphada, P.; Kadu, N.; Winter, K.; Sage, R. F.; Aguilar, C. N.; Schmutz, J.; Jenkins, J.; Holtum, J. A.; New Phytol. 2015, 207, 491.

5. Borland, A. M.; Griffiths, H.; Hartwell, J.; Smith, J. A. C.; J. Exp. Bot. 2009, 60, 279.

6. Davis, S. C.; LeBauer, D. S.; Long, S. P.; J. Exp. Bot. 2014, 65, 3471.

7. Kuloyo, O. O.; du Preez, J. C.; García-Apricio, M. P.; Kilian, S. G.; Steyn, L.; Gorgens, J.; World J. Microbiol. Biotechnol. 2014, 30, 3173. 
8. Lima, C. S. S.; Conceição, M. M.; Silva, F. L. H.; Lima, E. E.; Conrado, L. S.; Leão, D. A. S.; Appl. Energy. 2013, 102, 254.

9. Li, J.; Paul, M. C.; Younger, P. L.; Watson, I.; Hossain, M.; Welch, S.; Fuel 2014, 165, 205.

10. Williams, A.; Jones, J. M.; Ma, L.; Pourkashanian, M.; Prog. Energy Combust. Sci. 2012, 38, 113.

11. Capablo, J.; Salvado, J.; Renewable Energy 2017, 105, 449.

12. Saeed, U.; Rehman, M.; Applied Mathematics and Computation 2015 , 264, 310.

13. Souza, N.; Azevedo, J. L. T.; Fuel 2016, 185, 948.

14. Larsen, J.; Haven, M. O.; Thirup, L.; Biomass Bioenergy 2012, 46, 36.

15. Viikari, L.; Vehmaanpera, J.; Koivula, A.; Biomass Bioenergy 2012, 46, 13.

16. Bhutto, A. W.; Qureshi, K.; Harijan, K.; Abro, R.; Abbas, T.; Bazmi, A. A.; Karim, S.; Yu, G.; Energy 2017, 122, 724.

17. Mulakhudair, A. R.; Hanotu, J.; Zimmerman, W.; Biomass Bioenergy 2016, 93, 187

18. Cai, J.; He, Y.; Yu, X.; Banks, S. W.; Yang, Y.; Zhang, X.; Yu, Y.; Liu, R.; Bridgwater.; Renewable Sustainable Energy Rev. 2017, 76, 309.

19. Sluiter, A.; Ruiz, R.; Scarlata, C.; Sluiter, J.; Templeton, D.; Crocker, D.; National Renewable Energy Laboratory, Laboratory Analytical Procedure, 2008, disponível em https://www.nrel.gov/docs/gen/ fy13/42618.pdf, acessado em agosto 2018.

20. Rocha, G. J. M.; Silva, F. T.; Curvelo, A. A. S.; Araújo, G. T.; Resumos do $5^{\text {th }}$ Brazilian Symposium on the Chemistry of Lignins and Other Wood Components, Brasil, 1997.

21. Gouveia, E. R.; Nascimento, R. T.; Souto-Maior, A. M.; Rocha, G. J. M.; Quim. Nova 2009, 32, 1500.

22. Ghose, T. K.; Pure Appl. Chem. 1987, 59, 257.

23. Huang, Y. L.; Chow, C. J.; Fang, Y. J.; J. Food Drug Anal. 2011, 19, 318.

24. Pourbarani, M.; Forgács, G.; Horváth, I. S.; Niklasson, C.; Taherzadeh, M. J.; Bioresour. Technol. 2010, 101, 4246.

25. Meschede, D. K.; Velini, E. D.; Carbonari, C. A.; Moraes, C. P.; Planta Daninha 2012, 30, 121.
26. Tropea, A.; Wilson, D.; Torre, L. G. L.; Curto, R. B. L.; Saugman, P.; Troy-Davies, P.; Dugo, Giacomo.; Waldron, K. W.; J. Food Res. 2014, 30,60

27. Boonwong, T.; Karnnasuta, S.; Srinorakutara, T.; KKU Research Journal 2014, 19, 369 .

28. Chintagunta, A. D.; Ray, S.; Banerjee, R.; J. Cleaner Prod. 2017, 165, 1508.

29. Bhandari, S. V.; Panchapakesan, A.; Shankar, N.; Kumar, H. G. A.; International Journal of Scientific Research \& Technology 2013, 2, 362.

30. Niwaswong, C.; Chaiyamate, P.; Chotikosaijkanon, P.; Ruangviriyachai, C.; Afr. J. Biotechnol. 2014, 13, 3928.

31. Chintagunta, A. D.; Ray, S.; Banerjee, R.; J. Cleaner Prod. 2017, 165, 1508.

32. Gil, L. S.; Maupoey, P. F. J. Cleaner Prod. 2018, 172, 1224.

33. Demirbas, A.; Sahin, A.; Fuel Process. Technol. 1998, 55, 175.

34. Speight, J. G.; Synthetic Fuels Handbook: Properties, Process and Performance, McGraw-Hill Companies, Inc.: New York, 2008.

35. Romallosa, A. R. D.; Kraft, E.; Resources 2017, 6, 1.

36. Voicea, I.; Danciu, A.; Matache, M.; Voicu, G.; Vladut, V.; Ann. Fac. Eng. Hunedoara 2013, 11, 59.

37. Tumuru, J. S.; Sokhansanji, S.; Lim, C. J.; Bi, T.; Lau, A.; Melin, S.; Sowlati, T.; Oveisi, E.; Applied Engineering in Agriculture 2010, 26, 1013.

38. Adapa, P. K.; Schoe, G. J.; Tabil, L. G.; Sokhanasanj, S.; Crerar, B.; Pelleting of Fractionated Alfalfa Products, American Society of Associated Executives (ASAE): Washington, DC, 2003; pp. 104-115.

39. Olorunnisola, A.; Production of Fuel Briquettes from Waste Paper and Coconut Husk Admixtures, disponível em https://ecommons.cornell.edu/ handle/1813/10628, acessado em Agosto 2018.

40. Kentucky Educational Television, disponível em https://www.ket.org/ public-affairs/coal-advocate-makes-case-greenhouse-gas-regulations/, acessado em agosto 2018. 